\title{
Correction: Structure and dynamics of the pan- genome of Streptococcus pneumoniae and closely related species
}

\author{
Claudio Donati ${ }^{* *}$, N Luisa Hiller ${ }^{2}$, Hervé Tettelin ${ }^{3}$, Alessandro Muzzi ${ }^{1}$, Nicholas J Croucher ${ }^{4}$, Samuel V Angiuoli ${ }^{3}$, \\ Marco Oggioni ${ }^{5}$, Julie C Dunning Hotopp ${ }^{3}$, Fen Z Huㄹ, David R Riley ${ }^{3}$, Antonello Covacci ${ }^{1}$, Tim J Mitchell ${ }^{6}$, \\ Stephen D Bentley ${ }^{4}$, Mogens Kilian$^{7}$, Garth D Ehrlich ${ }^{2}$, Rino Rappuoli ${ }^{1}$, E Richard Moxon ${ }^{8}$ and Vega Masignani ${ }^{1}$
}

After publication of this Research [1], we noted that there is a misspelling in the name of Mogens Killian. Furthermore, we noted errors to Additional File 1, Table S1. For the strain S. pneumoniae AP200, the 'Location of isolation' should be 'Italy' rather than 'University of Siena'. Also, in the Acknowledgements, we should have thanked all the AP200 genome project participants for providing early access to the AP200 genome sequences rather than Annalisa Pantosti only. The AP200 genome project was supported by a grant from the Italian Ministry of Research (FIRB 2005-RBLA054453).

\footnotetext{
Author details

'Novartis Vaccines and Diagnostics, Via Fiorentina 1, 53100 Siena, Italy.

${ }^{2}$ Allegheny General Hospital, Allegheny-Singer Research Institute, Center for Genomic Sciences, Pittsburgh, Pennsylvania 152123, USA. Institute for Genome Sciences, Department of Microbiology and Immunology, University of Maryland School of Medicine, 801 West Baltimore Street, MD 21201, USA. ${ }^{4}$ The Sanger Institute, Wellcome Trust Genome Campus, Hinxton, Cambridge CB10 1SA, UK. ${ }^{5}$ Laboratorio di Microbiologia Molecolare e Biotecnologia, Dipartimento di Biologia Molecolare, Universita' di Siena, Policlinico Le Scotte, 53100 Siena, Italy. ${ }^{6}$ Division of Infection and Immunity, Glasgow Biomedical Research Centre, University of Glasgow, 120 University Place, Glasgow G12 8TA, UK. 'Institute of Medical Microbiology and Immunology, Aarhus University, DK-8000 Aarhus, Denmark. ${ }^{8}$ University of Oxford

Department of Paediatrics, Medical Sciences Division, John Radcliffe Hospital, Headington OX3 9DU, UK.
}

Received: 18 October 2011 Accepted: 20 October 2011

\section{Reference}

1. Claudio Donati, Luisa Hiller N, Hervé Tettelin, Alessandro Muzzi, Croucher JNicholas, Angiuoli VSamuel, Marco Oggioni, Dunning Hotopp CJulie, Hu ZFen, Riley RDavid, Antonello Covacci, Mitchell JTim, Bentley DStephen, Mogens Kilian, Ehrlich DGarth, Rino Rappuoli, Richard EMoxon, Vega Masignani: Structure and dynamics of the pangenome of Streptococcus pneumoniae and closely related species. Genome Biology 2010, 11:R107.

* Correspondence: claudio.donati@novartis.com

${ }^{1}$ Novartis Vaccines and Diagnostics, Via Fiorentina 1, 53100 Siena, Italy Full list of author information is available at the end of the article doi:gb-2011-12-10-140

Cite this article as: Donati et al:: Correction: Structure and dynamics of the pan-genome of Streptococcus pneumoniae and closely related species. Genome Biology 2011 12:140.
Submit your next manuscript to BioMed Central and take full advantage of:

- Convenient online submission

- Thorough peer review

- No space constraints or color figure charges

- Immediate publication on acceptance

- Inclusion in PubMed, CAS, Scopus and Google Scholar

- Research which is freely available for redistribution
Ciomed Central 